\title{
EVALUATING SCALE FUNCTIONS OF SPECTRALLY NEGATIVE LÉVY PROCESSES
}

\author{
B. A. SURYA, ${ }^{*}$ Bank of America
}

\begin{abstract}
In this paper we present a robust numerical method to compute the scale function $W^{(q)}(x)$ of a general spectrally negative Lévy process $(X, \mathrm{P})$. The method is based on the Esscher transform of measure $\mathrm{P}^{v}$ under which $X$ is taken and the scale function is determined. This change of measure makes it possible for the scale function to be bounded and, hence, makes numerical computation easy, fast, and stable. Working under the new measure $\mathrm{P}^{v}$ and using the method of Abate and Whitt (1992) and Choudhury, Lucantoni, and Whitt (1994), we give a fast stable numerical algorithm for the computation of $W^{(q)}(x)$.
\end{abstract}

Keywords: Lévy process; spectrally negative; scale function; Laplace transform.

2000 Mathematics Subject Classification: Primary 60G51; 62P05

Secondary 65T50

\section{Introduction}

In the literature we have seen that many fluctuation identities associated with the problem of first exit from the positive half-line or finite interval of a (reflected) spectrally negative Lévy process $(X, \mathrm{P})$ (a Lévy process with no positive jumps) can be written in terms of the so-called $q$-scale function $\left\{W^{(q)}(x): q \geq 0, x \in \mathbb{R}\right\}$. For a literature review, we refer the reader to [2], [5], [6], [18], [22], and the literature therein. In connection with the pricing of American put and Russian options driven by spectrally negative Lévy processes, the rational price of these options appears to be some functional of this function; see, for instance, [2]. In mathematical insurance this function appears in the problem of finding the optimal dividend payments; see, for instance, [3]. In credit risk theory the scale function plays an important role in determining the optimal endogenous default level as well as in assessing the optimality of the default level; see, for instance, [17]. Working under a completely general spectrally negative Lévy process, it was shown in [17] that not only is the analytical treatment of the optimal default level possible, but that the smooth-pasting condition used in [14] and [19] as the optimality criterion for choosing the optimal default level can be verified both analytically and numerically. It is also worth noting that the scale function appears to be an important factor in queueing theory (see, for instance, [10]) and the theory of continuous-state branching processes (we refer the reader to [15, Chapter 10] for more details).

For some spectrally negative Lévy processes, the scale function is available in explicit form. Typical examples are standard Brownian motions, $\alpha$-stable processes with $\alpha \in(0,2)$, jump diffusion processes with exponential negative jumps, compound Poisson processes, and so on. See, for instance, [5], [6], and [15] for further details. Owing to the complexity of the Laplace exponent of the Lévy process, the scale function is not available in explicit form in general.

Received 25 May 2007; revision received 26 November 2007.

* Postal address: Global Quantitative Risk Management, Quantitative Utility Department, Bank of America, N. A.,

9 Raffles Place \#15-00 Republic Plaza Tower 1, Singapore 048619.

Email address: budhi.a.surya@bankofamerica.com 
An example of this is a spectrally negative tempered stable process with index less than 2 . Thus, numerical inversion of the Laplace transform can be used to compute the scale function numerically. However, as shall be shown, it turns out that, for each $q>0$, the $q$-scale function $W^{(q)}(x)$ is exponentially unbounded under the measure $\mathrm{P}$ and, hence, numerical inversions for producing the $q$-scale function $W^{(q)}(x)$ could be unstable. This problem can be overcome by an appropriate choice of exponential change of measure $\mathrm{P}^{v}$ under which the scale function can be bounded. Working under the new measure $\mathrm{P}^{v}$ and using the method of [1] and [8], we give a fast stable numerical algorithm for the computation of the $q$-scale function $W^{(q)}(x)$ for all $q \geq 0$ of a general spectrally negative Lévy process.

The organization of this paper is as follows. In Section 2 we briefly discuss a spectrally negative Lévy process and its exponential change of measure $\mathrm{P}^{v}$. In Section 3 we define the $q$-scale function of a general spectrally negative Lévy process. Numerical inversion of the scale function is discussed in Section 4. Numerical examples of the computation of the scale function are given in Section 5. Finally, we provide the MATLAB ${ }^{\circledR}$ program code for the computation in Section 6.

\section{Spectrally negative Lévy processes}

In this paper we consider a Lévy process $X$ having the canonical decomposition

$$
X_{t}=\mu t+\sigma B_{t}+J_{t}^{(-)},
$$

where $B=\left\{B_{t}, t \geq 0\right\}$ is a standard Brownian motion and $J^{(-)}=\left\{J_{t}^{(-)}, t \geq 0\right\}$ is a nonGaussian Lévy process with no positive jumps and independent from $B$. This class of process is of great interest from the theoretical point of view because it classifies processes for which fluctuation theory takes the nicest form and can be developed explicitly to its full extent. The degenerate case when $X$ is either the negative of a subordinator or a deterministic drift is of no interest and will not be discussed in this paper. What we shall say here is based for the most part on Chapter VII of [4].

The law of the Lévy process started at 0 will be denoted by $\mathrm{P}$ (with the associated expectation operator $\mathrm{E})$. Since $X$ has no positive jumps, the moment generating function $\mathrm{E}\left(\exp \left(\theta X_{t}\right)\right)$ exists for all $\theta \geq 0$ and is given by

$$
\mathrm{E}\left(\exp \left(\theta X_{t}\right)\right)=\mathrm{e}^{t \kappa(\theta)},
$$

where the function $\kappa:[0, \infty) \rightarrow(-\infty, \infty)$, also called the Laplace exponent of $X$, is defined by

$$
\kappa(\theta)=\mu \theta+\frac{1}{2} \sigma^{2} \theta^{2}+\int_{(-\infty, 0)}\left(\mathrm{e}^{\theta y}-1-\theta y \mathbf{1}_{\{y>-1\}}\right) \Pi(\mathrm{d} y),
$$

where $\mu \in \mathbb{R}, \sigma \geq 0$, and $\Pi$ is a measure concentrated on $(-\infty, 0)$ satisfying $\int_{(-\infty, 0)}(1 \wedge$ $\left.y^{2}\right) \Pi(d y)<\infty$. It is easily seen that $\kappa$ is 0 at the origin and is strictly convex with $\lim _{\theta \uparrow \infty} \kappa(\theta)=\infty$. Next we denote by $\Phi(\alpha)$ the largest solution of the equation

$$
\kappa(p)=\alpha \quad \text { for a given } \alpha \geq 0 .
$$

Note that, owing to the convexity of $\kappa$, there exists at most two roots for a given $\alpha$ and precisely one root when $\alpha>0$. The asymptotic behavior of $X$ can be determined from the sign of $\kappa^{\prime}(0+)$, the right-derivative of $\kappa$ at 0 . The Lévy process $X$ drifts to $-\infty$, oscillates or drifts to $+\infty$ according to whether $\kappa^{\prime}(0+)$ is negative, 0 , or positive. We refer the reader to [16] for further discussions. 
It is worth mentioning that, under the measure $\mathrm{P}^{v}$ defined by

$$
\left.\frac{\mathrm{dP}}{\mathrm{dP}}\right|_{\mathcal{F}_{t}}=\exp \left(v X_{t}-\kappa(v) t\right) \quad \text { for all } v \geq 0
$$

the Lévy process $\left(X, \mathrm{P}^{v}\right)$ is still a spectrally negative Lévy process. The Laplace exponent of $X$ under the measure $\mathrm{P}^{v}$ has changed to

$$
\begin{aligned}
\kappa_{v}(\theta) & =\frac{1}{t} \log \mathrm{E}^{v}\left(\exp \left(\theta X_{t}\right)\right) \\
& =\frac{1}{t} \log \mathrm{E}\left(\exp \left((\theta+v) X_{t}-\kappa(v) t\right)\right) \\
& =\kappa(\theta+v)-\kappa(v) .
\end{aligned}
$$

To each $v \geq 0$, we will denote by $\mathrm{P}_{x}^{v}$ the translation of $\mathrm{P}^{v}$ under which $X_{0}=x$.

\section{Scale functions}

In this section we discuss the so-called scale functions. (See [4, Chapter VII], [5], and [6] for the origin of this function.) This function features invariably in all known identities for Laplace transforms of first-exit times and overshoots of (reflected) spectrally negative Lévy processes. See the aforementioned literature for details.

Definition 1. Let $q \geq 0$, and define $\Phi_{\nu}(q)$ as the largest root of the equation $\kappa_{v}(\theta)=q$.

Definition 2. ( $q$-scale function.) For a given spectrally negative Lévy process $X$ with Laplace exponent $\kappa(1)$, there exists, for every $q \geq 0$, a right-continuous function $W^{(q)}:[0, \infty) \rightarrow$ $[0, \infty)$, called the $q$-scale function, with Laplace transform given by

$$
\int_{0}^{\infty} \mathrm{e}^{-\lambda x} W^{(q)}(x) \mathrm{d} x=\frac{1}{\kappa(\lambda)-q} \quad \text { for } \lambda>\Phi(q),
$$

where $\Phi(q)$ is as defined in Section 2. We shall write for short $W^{(0)}=W$. Furthermore, we refer to $W_{v}^{(q)}(x)$, the scale function under the measure $\mathrm{P}^{v}$.

It turns out that the smoothness properties of the $q$-scale functions $W^{(q)}(x)$ are very closely related to the roughness of the paths of the associated Lévy process. If $X$ has a path of unbounded variation or bounded variation and the Lévy measure $\Pi$ has no atoms, it is known that the $q$-scale function $W^{(q)}(x)$ is continuously differentiable; see, for instance, [7], [18], and the literature therein for details.

Following (3), we see that if the $q$-scale function $W^{(q)}(x)$ of the Lévy process $(X, \mathrm{P})$ is computed by numerically inverting the right-hand side of (3) directly, it may cause instability in the computation, unless $q=0$ and the Laplace exponent $\kappa$ satisfies the condition $\kappa^{\prime}(0+)>0$. In order to avoid the problem of instability in the computation for the general case $(q \geq 0)$, we use the exponential change of measure $\mathrm{P}^{\Phi(q)}$ in (2) to first numerically compute the scale function $W_{\Phi(q)}(x)$, which plays the role of $W^{(0)}(x)$ when $X$ is taken under the measure $\mathrm{P}^{\Phi(q)}$. Using this result, we are able to produce the $q$-scale function $W^{(q)}(x)$ for $q \geq 0$. Proposition 1 , below, explains this further. 
Proposition 1. (Asymptotic behavior.) Suppose that either $q>0$ or $q=0$ and $\kappa^{\prime}(0+)>0$. Then the scale function $\left\{W_{\Phi(q)}(x): q \geq 0, x \in \mathbb{R}\right\}$ of $X$ taken under the measure $\mathrm{P}^{\Phi(q)}$ is increasing and bounded by $1 / \kappa^{\prime}(\Phi(q))$, i.e.

$$
\lim _{x \rightarrow \infty} W_{\Phi(q)}(x)=\frac{1}{\kappa^{\prime}(\Phi(q))} .
$$

However, when $X$ is taken under the measure $\mathrm{P}$, the $q$-scale function $W^{(q)}(x)$ is given by

$$
W^{(q)}(x)=\mathrm{e}^{\Phi(q) x} W_{\Phi(q)}(x),
$$

and, hence, has the asymptotic

$$
W^{(q)}(x) \sim \frac{\mathrm{e}^{\Phi(q) x}}{\kappa^{\prime}(\Phi(q))} \text { as } x \rightarrow \infty .
$$

By adapting arguments from the proof of Lemma 4(i) of [21], the statement of Proposition 1 can be justified.

Remark 1. In the case in which $q=0$ and $\kappa^{\prime}(0+)=0$ (hence, $\Phi(q)=0$ ) the scale function $W_{\Phi(q)}(x)$ is no different from the $q$-scale function $W^{(q)}(x)$ and is unbounded at $\infty$, i.e.

$$
\lim _{x \rightarrow \infty} W_{\Phi(q)}(x)=\infty .
$$

(For a numerical illustration of this case, see, for instance, Figures 7 and 8 in Section 5.)

Remark 2. Following (3), it is straightforward to check that

$$
W_{v}^{(q)}(x)=\mathrm{e}^{-v x} W^{(q+\kappa(v))}(x) \text { for all } v \geq 0 \text { and } q \geq-\kappa(v) .
$$

To verify relation (4), apply a Laplace transform to both sides.

\section{Numerical inversions of the scale function}

In this section we discuss the numerical inversion of the Laplace transform

$$
\int_{0}^{\infty} \mathrm{e}^{-\lambda x} W_{\Phi(q)}(x) \mathrm{d} x=\frac{1}{\kappa(\lambda+\Phi(q))-q} \text { for } \lambda>0 \text { and } q \geq 0,
$$

for the computation of the scale function $W_{\Phi(q)}(x)$ of the Lévy process $\left(X, \mathrm{P}^{\Phi(q)}\right)$. The result is used to compute the $q$-scale function

$$
\left\{W_{\nu}^{(q)}(x): v \geq 0, q \geq-\kappa(v), x \in \mathbb{R}_{+}\right\}
$$

of the Lévy process $\left(X, \mathrm{P}^{v}\right)$. To deal with a general class of spectrally negative Lévy processes, we present an algorithm based on the method of [1] and [8] here.

To start with, let $f$ be a real-valued function defined on the positive real-line (not necessarily a probability density). For such a function $f$, it is often convenient to work with the Laplace transform

$$
\hat{f}(\lambda)=\int_{0}^{\infty} \mathrm{e}^{-\lambda x} f(x) \mathrm{d} x
$$


where $\lambda$ is a complex variable for which the integral exists. The standard inversion integral for the Laplace transform $\hat{f}$ is the Bromwich contour integral, which can also be expressed as the integral of a real-valued function of a real variable by choosing a specific contour to be any vertical line $\lambda=a_{1}$ such that $\hat{f}(\lambda)$ has no singularities on or to the right of the vertical line. By applying this integral we obtain

$$
f(x)=\frac{1}{2 \pi \mathrm{i}} \int_{a_{1}-\mathrm{i} \infty}^{a_{1}+\mathrm{i} \infty} \mathrm{e}^{\lambda x} \hat{f}(\lambda) \mathrm{d} \lambda=\frac{\exp \left(a_{1} x\right)}{2 \pi} \int_{-\infty}^{\infty} \mathrm{e}^{\mathrm{i} \lambda x} \hat{f}\left(a_{1}+\mathrm{i} \lambda\right) \mathrm{d} \lambda .
$$

For some Laplace transforms $\hat{f}$, analytic expressions for $f$ are available explicitly; see, for instance, [20]. When the transform cannot be inverted analytically, the function $f$ can be approximated by means of a numerical approximation.

Several numerical inversion algorithms have been proposed by several authors. The fast and stable one is given in [1] and [8]. Following [1], we use the trapezoidal rule to approximate the integral in (6) and analyze the corresponding discretization error using the Poisson summation formula. The trapezoidal rule approximates the integral of a function $g$ over the bounded interval $[c, d]$ by the integral of the piecewise linear function obtained by connecting the $n+1$ evenly spaced points $g(c+k h), 0 \leq k \leq n$, where $h=(d-c) / n$. Using the trapezoidal rule, we have

$$
\int_{c}^{d} g(x) \mathrm{d} x \approx h\left(\frac{g(c)+g(d)}{2}+\sum_{k=1}^{n-1} g(c+k h)\right)
$$

see [9, p. 51]. The trapezoidal rule (7) also applies for the case in which $c=-\infty$ and $d=\infty$ with the following modification

$$
\int_{-\infty}^{\infty} g(x) \mathrm{d} x \approx h_{1} \sum_{j=-\infty}^{\infty} g\left(j h_{1}\right)
$$

where $h_{1}$ is a small positive constant. Applying (8) to (6) with a step size $h_{1}=\pi / x, x>0$, and letting $a_{1}=A_{1} / x$ at the same time, we obtain

$$
f(x) \approx \frac{\exp \left(A_{1}\right)}{2 x} \sum_{j=-\infty}^{\infty}(-1)^{j} \hat{f}\left(\frac{A_{1}+\mathrm{i} j \pi}{x}\right) .
$$

The advantage of using the simple numerical procedure of the trapezoidal rule is that it tends to work well for periodic and oscillating integrands since the errors tend to cancel out and the realized errors can be substantially less than from other alternative numerical procedures such as Simpson's rule. Moreover, the Poisson summation formula can be applied to obtain a convenient representation of the discretization error associated with the trapezoidal rule. Using the Poisson summation formula, approximation (9) can be obtained for an integrable function $g$ by

$$
\sum_{j=-\infty}^{\infty} g\left(x+\frac{2 \pi j}{h_{2}}\right)=\frac{h_{2}}{2 \pi} \sum_{j=-\infty}^{\infty} \exp \left(-\mathrm{i} j h_{2} x\right) \phi\left(j h_{2}\right),
$$

where $h_{2}$ is some positive constant and

$$
\phi(u)=\int_{-\infty}^{\infty} \mathrm{e}^{\mathrm{i} u x} g(x) \mathrm{d} x
$$


the Fourier transform of $g$. In order to control the aliasing error (aliasing means that the new function is constructed by adding a translated version of the original function), we use exponential damping; that is, if $f$ is our original function of interest then we replace $g(x)$ by the function $f(x) \exp \left(-a_{1} x\right) \mathbf{1}_{[0, \infty)}(x)$. Then

$$
\phi(\lambda)=\hat{f}\left(a_{1}-\mathrm{i} \lambda\right),
$$

and the right-hand side of (10) can be expressed in terms of Laplace transform values:

$$
\sum_{j=0}^{\infty} \exp \left(-a_{1}\left(x+\frac{2 \pi j}{h_{2}}\right)\right) f\left(x+\frac{2 \pi j}{h_{2}}\right)=\frac{h_{2}}{2 \pi} \sum_{j=-\infty}^{\infty} \exp \left(-\mathrm{i} j h_{2} x\right) \hat{f}\left(a_{1}-\mathrm{i} j h_{2}\right) .
$$

In addition, if we let $h_{2}=\pi / x l_{1}$ with $l_{1} \geq 1$ and $a_{1}=A_{1} / 2 x l_{1}$ in (11), we obtain

$$
f(x)=\frac{\exp \left(A_{1} / 2 l_{1}\right)}{2 x l_{1}} \sum_{j=-\infty}^{\infty} \exp \left(-\frac{i j \pi}{l_{1}}\right) \hat{f}\left(\frac{A_{1}}{2 x l_{1}}-\frac{i j \pi}{l_{1} x}\right)-\bar{e}_{\infty},
$$

where the error $\bar{e}_{\infty}$ is given by

$$
\bar{e}_{\infty}=\sum_{j=1}^{\infty} \exp \left(-2 j A_{1}\right) f((2 j+1) x) .
$$

Comparing (11) with (9), we conclude that $\bar{e}$ is an explicit expression for the discretization error associated with the trapezoidal rule approximation. This discretization error can be bounded easily whenever $f$ is bounded. For example, if $|f(x)| \leq C$ for some positive constant $C$ and all $x \geq 0$ then we have

$$
\left|\bar{e}_{\infty}\right| \leq \frac{C \exp \left(-2 A_{1}\right)}{\left(1-\exp \left(-2 A_{1}\right)\right)} .
$$

(If $f(x)$ is the scale function $W_{\Phi(q)}(x)$ then $C=1 / \kappa^{\prime}(\Phi(q))$. If $f(x)$ is the probability distribution then $C=1$.) Therefore, an approximation of the function $f$ in (6) is given by

$$
S_{N}(x)=\frac{\exp \left(A_{1} / 2 l_{1}\right)}{2 x l_{1}} \sum_{j=-N}^{N} \exp \left(-\frac{i j \pi}{l_{1}}\right) \hat{f}\left(\frac{A_{1}}{2 x l_{1}}-\frac{i j \pi}{l_{1} x}\right) .
$$

The raw value of $S_{N}$ may not be a very good approximation, but, by using Euler's summation to smooth the values of the (nearly) alternating sums we were able to obtain good accuracy. The approximation to $f(x)$ is finally given by

$$
f(x) \doteqdot \sum_{n=0}^{M} 2^{-M}\left(\begin{array}{c}
M \\
n
\end{array}\right) S_{N+n}(x) .
$$

This is the formula that we are going to use in this paper to compute the scale function $W_{\Phi(q)}(x)$ by numerically inverting its Laplace transform given by (5). 


\section{Numerical examples}

For our numerical examples, we consider four different Lévy processes. Firstly, we assume that $X$ is generated by $\alpha$-stable processes with Laplace exponent

$$
\kappa(\lambda)=K \lambda-\lambda^{\alpha} \quad \text { for } \alpha \in(0,1) \quad \text { and } \quad \kappa(\lambda)=K \lambda^{\alpha} \quad \text { for } \alpha \in[1,2],
$$

for some constants $K$. Secondly, we consider jump diffusion processes where the jump component of $X$ is generated by a compound Poisson process having independent downward jumps with exponential $\mathrm{e}^{b}$ distribution occurring at the times of a Poisson process with rate $a$; that is to say that $X$ has Laplace exponent

$$
\kappa(\lambda)=d \lambda+\frac{\sigma^{2}}{2} \lambda^{2}-\frac{a \lambda}{b+\lambda} .
$$

Thirdly, we consider $X$ to be a one-sided tempered stable process. In the general case (i.e. $\alpha \neq 1$ and $\alpha \neq 0$ ) the Laplace exponent of $X$ is given by

$$
\kappa(\lambda)=d \lambda+\Gamma(-\alpha) \beta^{\alpha} C\left(\left(1+\frac{\lambda}{\beta}\right)^{\alpha}-1-\frac{\lambda \alpha}{\beta}\right) .
$$

Finally, we consider the case where $X$ is a gamma process perturbed by a diffusion process. The Laplace exponent of $X$ is described by

$$
\kappa(\lambda)=d \lambda+\frac{\sigma^{2}}{2} \lambda^{2}-\psi(\lambda)
$$

where $\psi(\lambda)$ is the Laplace exponent of a gamma process $S$ given by

$$
\psi(\lambda)=a \log \left(1+\frac{\lambda}{b}\right)=\int_{0}^{\infty}\left(1-\mathrm{e}^{-\lambda x}\right) a x^{-1} \mathrm{e}^{-b x} \mathrm{~d} x \quad \text { for } a, b>0 .
$$

Note that the perturbed gamma process (16) is a slight generalization of the model studied in [11] and has been used in risk theory quite extensively. For related references, see, for instance, [11]-[13], [23], and the literature therein for details.

For all computations, we fix $N=11, M=9, A_{1}=14.0$, and $l_{1}=1$; for the jump diffusion and gamma processes, we set $\sigma=0.2, d=0.055, a=0.5$, and $b=9$; for the $\alpha$-stable process, we set $K=0.5$ for $\alpha=2$ (a standard Brownian motion) and $K=1$ for $\alpha=1.75$. In the case where $X$ is a tempered stable process with $\alpha=0.5$ or $\alpha=1.5$ we choose the relative frequency of downward jumps $C$ to be 0.075 or 0.05 , respectively, and the jump rate $\beta$ to be 2.5. The numerical results of the scale function $W_{\Phi(q)}(x)$ in (5) for $q=0.1$ are presented in Figures 1-6. We observe from these plots that all of the curves are increasing and bounded from above by $1 / \kappa^{\prime}(\Phi(q))$. These numerical outcomes support the previous theoretical results stated in Proposition 1. In particular, for the case where $X$ has a path of bounded and unbounded variation we respectively see that

$$
W_{\Phi(q)}(0)>0 \quad \text { and } \quad W_{\Phi(q)}(0)=0 ;
$$

see [17, Section 4] for further properties of the scale function at 0 .

Using the explicit form of the scale function $W_{\Phi(q)}(x)$ of $\alpha$-stable (13) and jump diffusion (14) processes, given in the appendix of [17], we present in Figures 2 and 4 plots of the 
absolute error between the theoretical curve $W_{\Phi(q)}(x)$ and the numerical curve $\hat{W}_{\Phi(q)}(x)$ produced by the proposed numerical method. Following (12), we observe that the absolute error $\left|\hat{W}_{\Phi(q)}(x)-W_{\Phi(q)}(x)\right|$ is bounded from above by a constant

$$
\frac{1}{\kappa^{\prime}(\Phi(q))} \frac{\exp \left(-A_{1}\right)}{\left(1-\exp \left(-A_{1}\right)\right)}
$$

i.e.

$$
\left|\hat{W}_{\Phi(q)}(x)-W_{\Phi(q)}(x)\right| \leq \frac{1}{\kappa^{\prime}(\Phi(q))} \frac{\exp \left(-A_{1}\right)}{\left(1-\exp \left(-A_{1}\right)\right)} .
$$

Since the (tuning) parameter $A_{1}$ was chosen to be relatively big (with $A_{1}=14$ ), we see that the absolute error is relatively small and, hence, our numerical method performs well in the computation of the scale function $W_{\Phi(q)}(x)$.

Figures 5 and 6 show the shape of the scale function $W_{\Phi(q)}(x)$ for tempered stable processes (15) with indices $\alpha=0.5$ and $\alpha=1.5$, and for a perturbed gamma process (16), respectively. For these stochastic processes, we note that an explicit expression for the scale function $W_{\Phi(q)}(x)$ is not available. But, nonetheless, it exhibits the important properties of the scale function, as specified in Proposition 1 and those found in, among others, [7], [15], [17], and [21].

Figures 7 and 8 respectively display the shape of the scale function $W_{\Phi(q)}(x)$ and the estimation error for an $\alpha$-stable process (13) for $q=0$ and index $\alpha=1.5$. From Figure 7 we see that the scale function is not bounded at large values of $x$. This observation is due to the fact that, for this process, we have $\kappa^{\prime}(0+)=0$ (the process oscillates). The estimation error as seen in Figure 8 is relatively small, however, it is not bounded at large values of $x$.

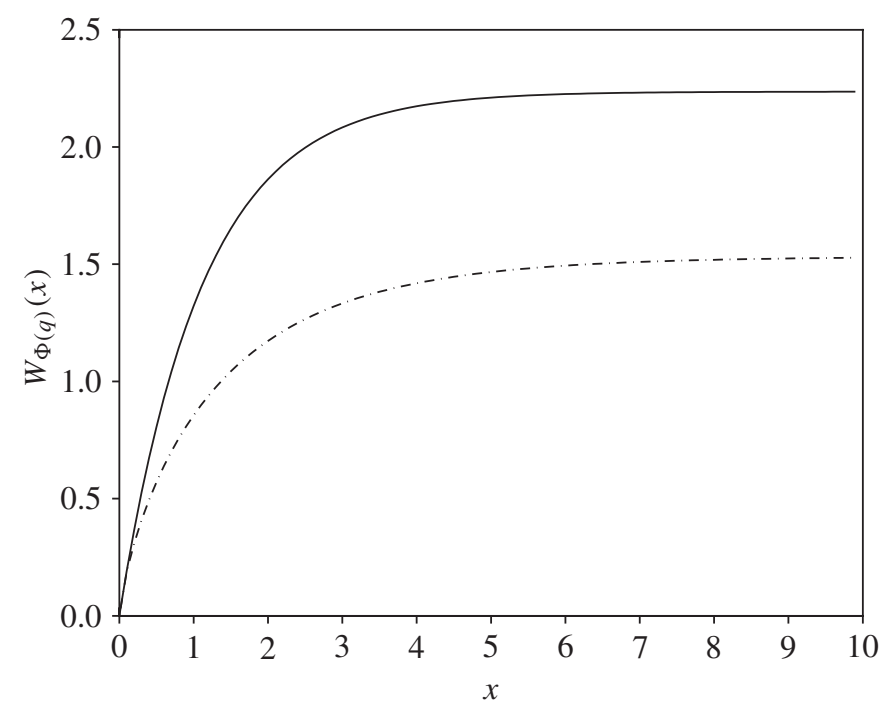

Figure 1: The shape of $W_{\Phi(q)}(x)$ for $\alpha$-stable Lévy processes with index $\alpha=2$ (solid line) and $\alpha=1.75$ (dash-dot line). For $\alpha=2$ and $\alpha=1.75$, the curve is increasing and bounded by $1 / \kappa^{\prime}(\Phi(q))=2.2361$ and 1.5530 , respectively. 


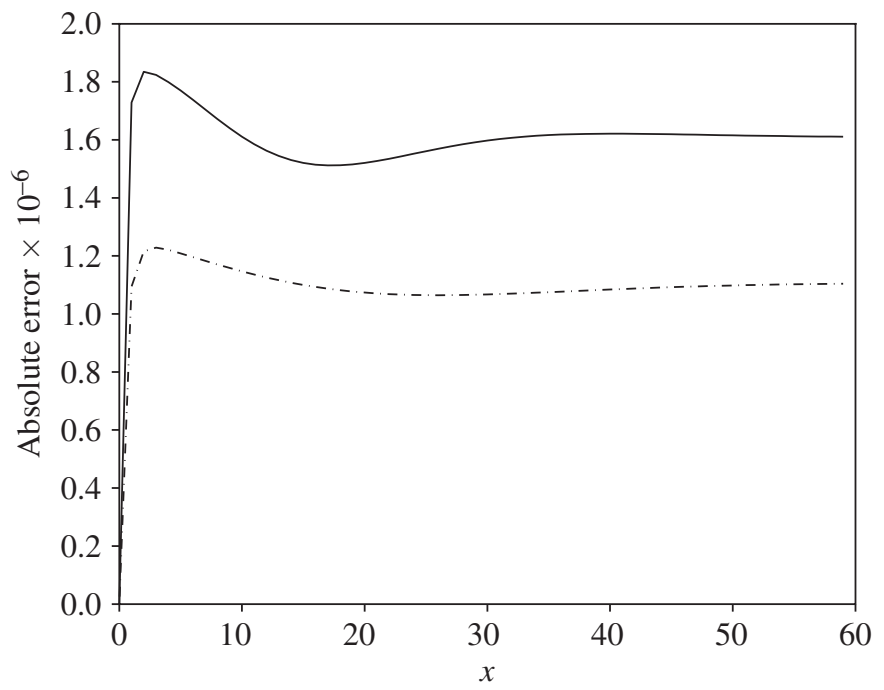

FIGURE 2: The absolute error $\left|\hat{W}_{\Phi(q)}(x)-W_{\Phi(q)}(x)\right|$ for $\alpha$-stable Lévy processes with index $\alpha=2$ (solid line) and $\alpha=1.75$ (dash-dot line). For $\alpha=2$ and $\alpha=1.75$, the error is bounded by $1.8594 \times 10^{-6}$ and $1.2747 \times 10^{-6}$, respectively. See (17) for the error estimate.

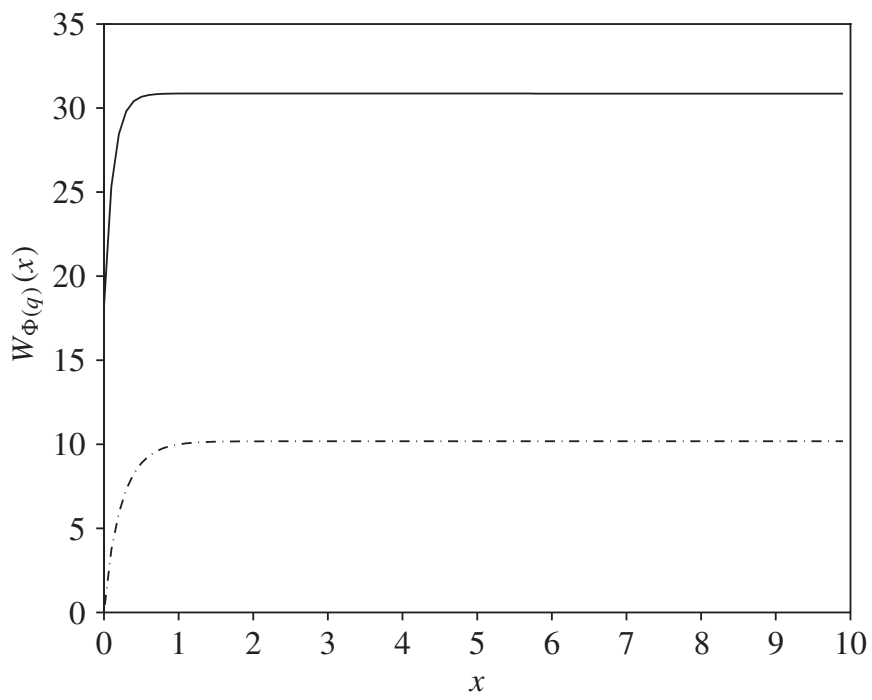

FIGURE 3: The shape of $W_{\Phi(q)}(x)$ for a compound Poisson process (solid line) and a jump diffusion process (dash-dot line). For the compound Poisson process and the jump diffusion process, the curve is increasing and bounded by $1 / \kappa^{\prime}(\Phi(q))=30.8640$ and 10.1787 , respectively. 


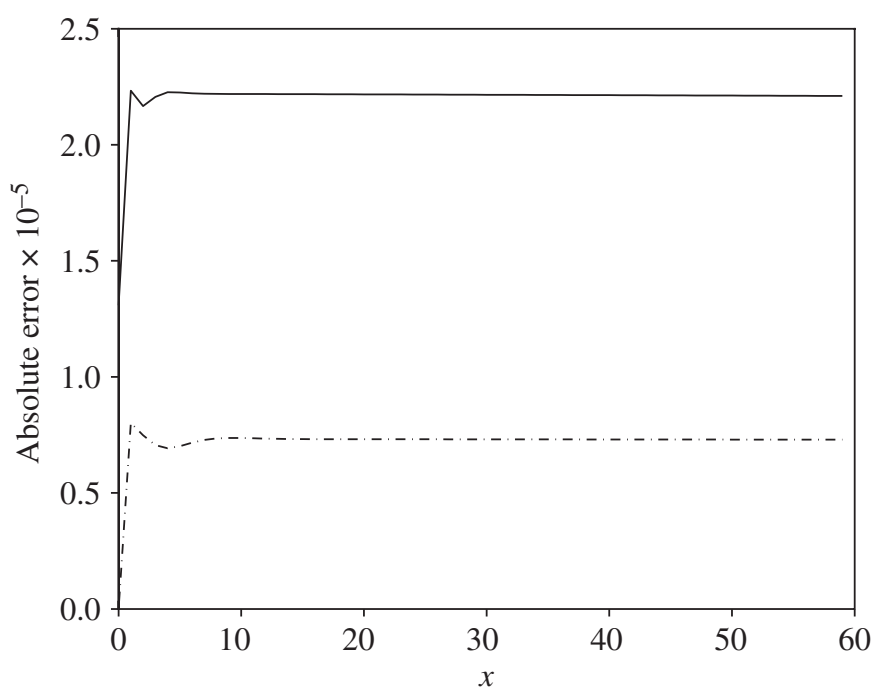

FIGURE 4: The absolute error $\left|\hat{W}_{\Phi(q)}(x)-W_{\Phi(q)}(x)\right|$ for a compound Poisson process (solid line) and a jump diffusion process (dash-dot line). For the compound Poisson process and the jump diffusion process, the error is bounded by $2.5664 \times 10^{-5}$ and $8.4639 \times 10^{-6}$, respectively. See (17) for the error estimate.

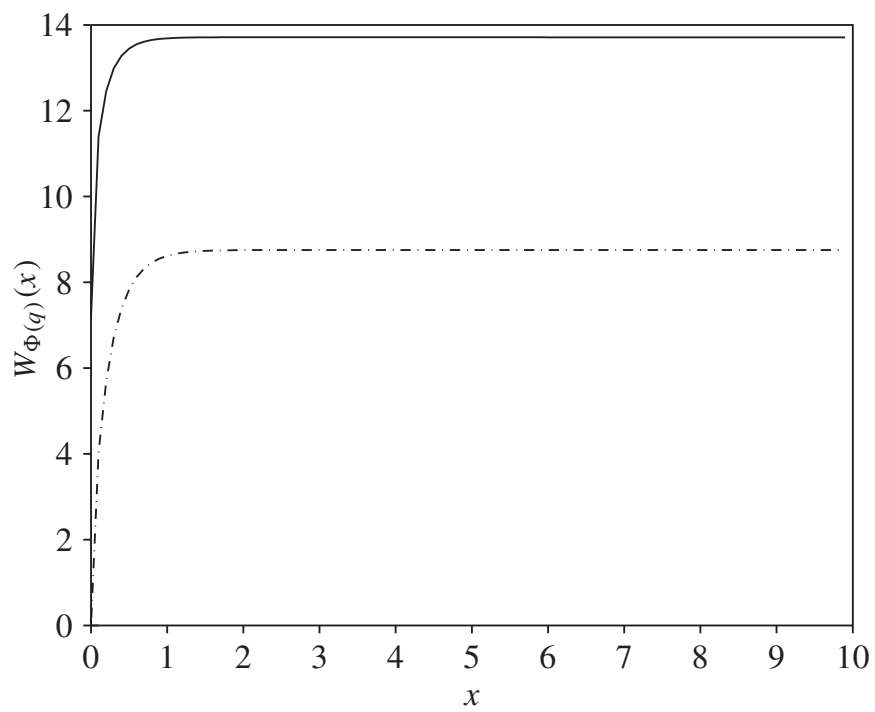

FIGURE 5: The shape of $W_{\Phi(q)}(x)$ for tempered stable Lévy processes with index $\alpha=0.5$ (solid line) and $\alpha=1.5$ (dash-dot line). For $\alpha=0.5$ and $\alpha=1.5$, the curve is increasing and bounded by $1 / \kappa^{\prime}(\Phi(q))=13.7137$ and 8.7532 , respectively. 


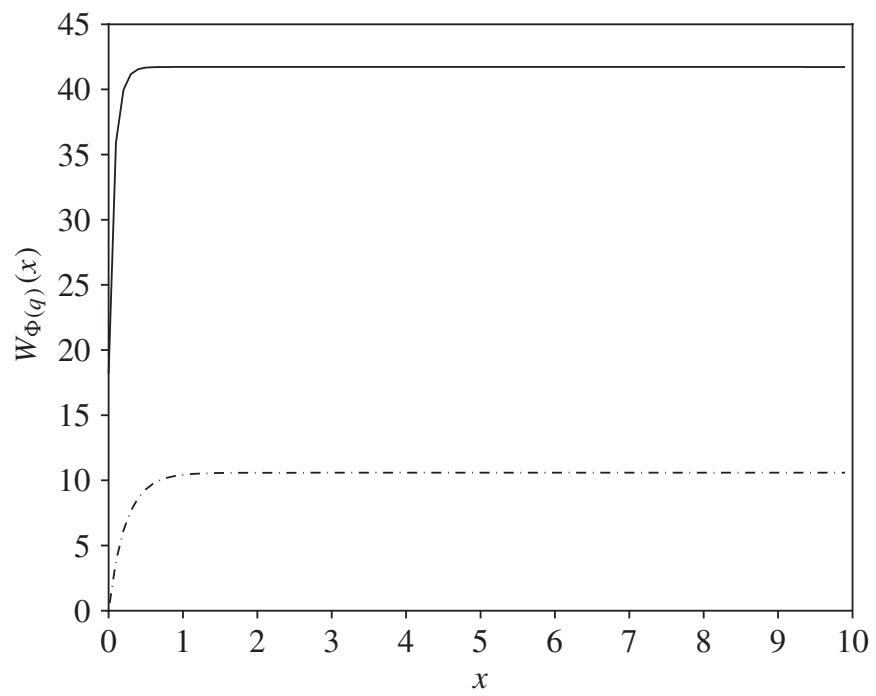

FIGURE 6: The shape of $W_{\Phi(q)}(x)$ for gamma processes perturbed by a diffusion process with $\sigma>0$ (dash-dot line) and $\sigma=0$ (solid line). For $\sigma>0$ and $\sigma=0$, the curve is increasing and bounded by $1 / \kappa^{\prime}(\Phi(q))=10.5823$ and 41.7222 , respectively.

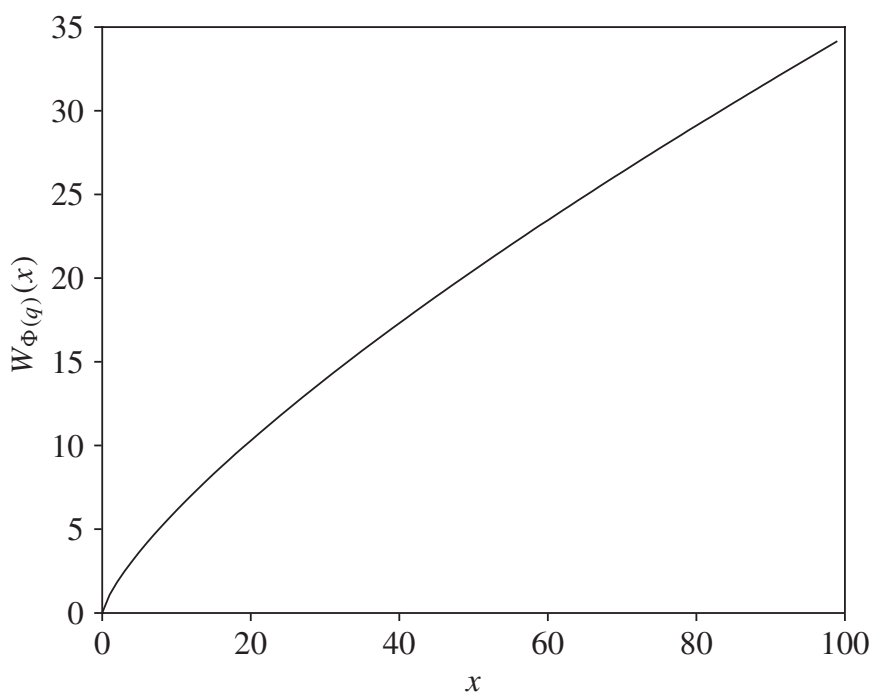

FiguRE 7: The shape of $W_{\Phi(q)}(x)$ for an $\alpha$-stable Lévy process for $q=0$ and index $\alpha=1.75$. In this case $\kappa^{\prime}(0+)=0$. From the plot we note that the curve is increasing and not bounded. 


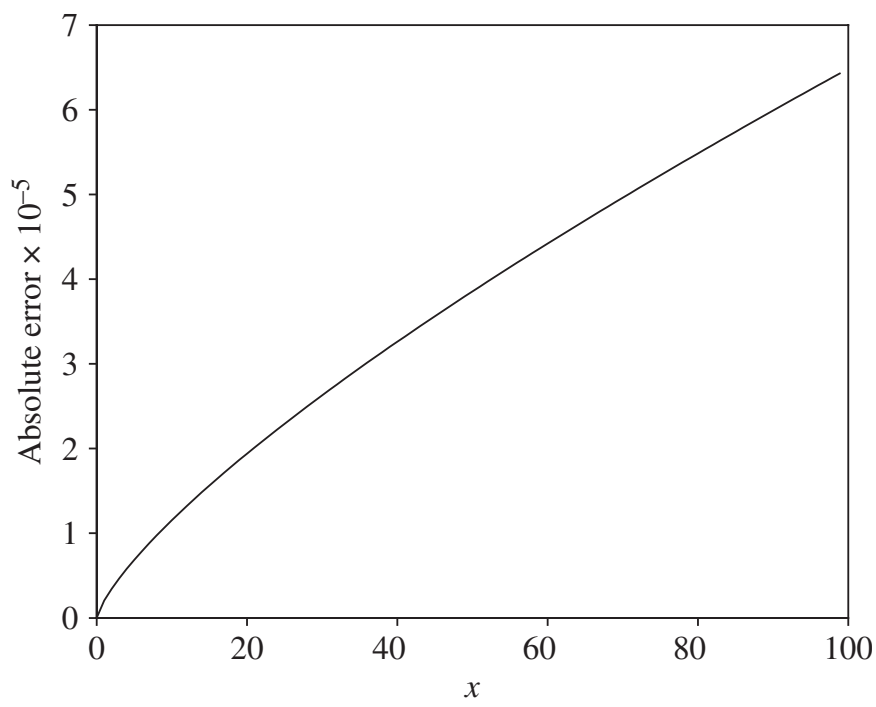

FIGURE 8: The absolute error $\left|\hat{W}_{\Phi(q)}(x)-W_{\Phi(q)}(x)\right|$ for an $\alpha$-stable Lévy process for $q=0$ and index $\alpha=1.75$. From the plot we note that the absolute error is not bounded.

\section{MATLAB program code}

In this section we present MATLAB codes used to compute $W_{\Phi(q)}(x)$ by numerically inverting the Laplace transform (5). The algorithm is based on the framework described in Section 4. To handle matrix multiplication, we use the approach of [22].

function $\mathrm{G}=\operatorname{ILT}(\mathrm{F}, \mathrm{X}, \mathrm{P})$

\% This program computes the scale function by numerically

\% inverting the univariate Laplace transform. Here, $F$ is the

\% Laplace transform function and $\mathrm{P}$ denotes the parameters

\% sitting in F. Setting the parameter values $11, \mathrm{~N}, \mathrm{M}$, and $\mathrm{A} 1$ :

$\mathrm{N}=11 ; \mathrm{M}=9 ; \mathrm{A} 1=14.0 ; \quad 11=1 ;$

\% Creating the weights to be used in the Euler summation

\% of the partial sums:

$\mathrm{mx}=\mathrm{pascal}(\mathrm{M}+1) ; \mathrm{my}=\mathrm{fliplr}(\mathrm{mx}) ; \operatorname{bn}=\operatorname{diag}(\mathrm{my}) * 2^{\wedge}(-\mathrm{M})$;

weight=ones $\left(\left[2{ }^{*} \mathrm{~N}+1\right.\right.$ 1]); head=cumsum(bn); tail=1-cumsum(bn) ;

$\operatorname{tail}(\mathrm{M}+1,:)=[]$; head $(\mathrm{M}+1,:)=[]$; weight=[head;weight; tail] ;

\% Setting the values of the arguments at which the transform

\% series is to be evaluated:

$\operatorname{val1}=-(\mathrm{N}+\mathrm{M}):(\mathrm{N}+\mathrm{M}) ; \operatorname{val1}=\left(\mathrm{i}{ }^{*} \mathrm{pi}{ }^{*} \operatorname{val1}+\mathrm{A} 1 / 2\right) / 11 ; \mathrm{X} \_\mathrm{inv}=1 . \mathrm{X}$;

X_args $=$ kron (X_inv, val1); 
\% Evaluating the integrand at all the points:

integrand $=f e v a l\left(F, X \_a r g s, P\right) \ldots$

. ${ }^{*} \exp \left(\mathrm{X} \_\operatorname{args} * \operatorname{diag}(\operatorname{kron}(\mathrm{X}, \operatorname{ones}(1,1+2 * \mathrm{~N}+2 * \mathrm{M})))\right)$;

\% Preparing the matrix which will post multiply the integrand:

right=kron (diag (X_inv), weight $) /(2 * 11)$;

\% Finally, the answer is given by

G=real (integrand*right) ;

The following MATLAB m-files are needed to compute, using (5), the scale function $W_{\Phi(q)}(x)$ of $\alpha$-stable, jump diffusion, tempered stable, and gamma processes whose Laplace exponents are given in (13)-(16), respectively.

function $g=\operatorname{LEXP}(z, A)$

\% The nonzero value of parameter $A$ is needed to compute the

\% largest root of the Laplace exponent. For spectrally

\% negative (SN) alpha-stable processes with alpha<1,

alpha=0.5; K=1; f=K. *z-z.^(alpha); $g=f-A ;$

\% For SN alpha-stable processes with alpha>=1,

alpha=1.75; K=1; $\mathrm{f}=\mathrm{K} \cdot{ }^{*} \mathrm{z} \cdot{ }^{\wedge}(\mathrm{alpha}) ; \mathrm{g}=\mathrm{f}-\mathrm{A}$;

\% For SN alpha-stable Levy processes with alpha=2,

$\mathrm{f}=\mathrm{z} \cdot{ }^{\wedge} 2 / 2 ; g=f-\mathrm{A} ;$

\% For SN jump diffusion processes,

sigma $=0.2 ; \quad a=0.5 ; \quad c=9 ; \quad d=0.055 ;$

$\mathrm{f}=\mathrm{d} \cdot{ }^{*} \mathrm{z}+0.5 \cdot{ }^{*}\left(\operatorname{sigma} \cdot{ }^{\wedge} 2\right) \cdot *\left(\mathrm{z} \cdot{ }^{\wedge} 2\right)-\mathrm{a} \cdot{ }^{*} \mathrm{z} \cdot /(\mathrm{c}+\mathrm{z}) ; \mathrm{g}=\mathrm{f}-\mathrm{A}$;

\% For SN bounded variation tempered stable processes,

alpha $=0.5 ;$ beta $=2.5 ; \mathrm{b}=0.075 ; \mathrm{d}=0.0550$;

\% For SN unbounded variation tempered stable processes,

alpha=1.5; beta $=2.5 ; \mathrm{c}=0.05 ; \mathrm{d}=0.0550$;

$\mathrm{f}=\mathrm{d} . * \mathrm{z}+\mathrm{gamma}(-\mathrm{alpha}) \cdot{ }^{*}($ beta $) \cdot{ }^{\wedge}(\mathrm{alpha}) \cdot *(\mathrm{C}) \ldots$

.* ((1+z./(beta)).^(alpha)-1-(alpha) .*z./beta); $g=f-A ;$ 
\% For gamma processes perturbed by a diffusion process,

sigma $=0.2 ; d=0.0550 ; a=0.5 ; b=9 ;$

$\mathrm{f}=\mathrm{d} \cdot{ }^{*} \mathrm{z}+0.5 \cdot{ }^{*}\left(\operatorname{sigma} \cdot{ }^{\wedge} 2\right) \cdot{ }^{*}\left(\mathrm{z} \cdot{ }^{\wedge} 2\right)-\mathrm{a} \cdot{ }^{*} \log (1+\mathrm{z} \cdot / \mathrm{b}) ; \mathrm{g}=\mathrm{f}-\mathrm{A}$;

function $\mathrm{f}=\operatorname{Phir}(\mathrm{q})$

\% Computing the largest root of the Laplace exponent:

$\mathrm{x} 0=1 ; \mathrm{f}=\mathrm{fsolve}\left({ }^{\prime} \mathrm{LEXP}\right.$ ', $\mathrm{x} 0$, optimset ('MaxFunEvals' , 100), q) ;

function f=funcscale (lambda, q)

\% The right-hand side of (5):

$\mathrm{A}=0 ; \mathrm{g}=(\operatorname{LEXP}(\operatorname{lambda}+\operatorname{Phir}(\mathrm{q}), \mathrm{A})-\mathrm{q}) ; \mathrm{f}=1 . / \mathrm{g} ;$

The scale function $W_{\Phi(q)}(x)$ is given by the following algorithm.

function $W=\operatorname{Scale}(x, q)$

\% Producing the scale function:

$\mathrm{W}=\operatorname{ILT}\left(\right.$ ' funcscale' $\left.^{\prime} \mathrm{x}, \mathrm{q}\right)$;

Having computed the function $W_{\Phi(q)}(x)$, the $q$-scale function $W^{(q)}(x)$ is finally given by the following algorithm.

function $\mathrm{W}=\mathrm{qSCale}(\mathrm{x}, \mathrm{q})$

\% Producing the q-scale function:

$\mathrm{W}=\exp \left(\operatorname{Phir}(\mathrm{q}){ }^{*} \mathrm{x}\right) \cdot{ }^{*} \operatorname{Scale}(\mathrm{x}, \mathrm{q})$;

\section{Acknowledgements}

This paper is based on a chapter of the author's Doctoral Thesis written under the supervision of Andreas Kyprianou and Richard Gill to whom the author acknowledges their very useful comments. The author is also grateful to a number of anonymous referees and associate editors for their useful suggestions that improved the presentation of this paper.

\section{References}

[1] Aвate, J. And Whitt, W. (1992). The Fourier-series method for inverting transform of probability distributions. Queuing Systems Theory Appl. 10,5-87.

[2] Avram, F., Kyprianou, A. E. and Pistorius, M. R. (2004). Exit problems for spectrally negative Lévy processes and applications to Russian, American and Canadized options. Ann. Appl. Prob. 14, 215-238.

[3] Avram, F., Palmowski, Z. and Pistorius, M. R. (2006). On the optimal dividend problem for a spectrally negative Lévy process. Ann. Appl. Prob. 17, 156-180.

[4] Bertoin, J. (1996). Lévy Processes. Cambridge University Press.

[5] Bertoin, J. (1996). On the first exit-time of a completely asymmetric stable process from finite interval. Bull. London Math. Soc. 5, 514-520. 
[6] Bertoin, J. (1997). Exponential decay and ergodicity of completely asymmetric Lévy processes in a finite interval. Ann. Appl. Prob. 7, 156-169.

[7] Chan, T. and Kyprianou, A. E. (2007). Smoothness of scale functions for spectrally negative Lévy processes. Preprint.

[8] Choudhury, G. L., Lucantoni, D. M. and Whitt, W. (1994). Multidimensional transform inversion with applications to the transient M/G/1 queue. Ann. Appl. Prob. 4, 719-740.

[9] Davis, P. J. And Rabinowitz, P. (1984). Methods of Numerical Integration, 2nd edn. Academic Press, New York.

[10] Dube, P., Guillemin, F. and Mazumdar, R. (2004). Scale functions of Lévy processes and busy periods of finite capacity. J. Appl. Prob. 41, 1145-1156.

[11] Dufresne, F. And Gerber, H. U. (1993). The probability of ruin for the inverse Gaussian and related processes. Insurance Math. Econom. 12, 9-22.

[12] Gerber, H. U. (1992). On the probability of ruin for infinitely divisible claim amount distributions. Insurance Math. Econom. 11, 163-166.

[13] Gerber, H. U. AND Shiu, E. S. W. (1994). Martingale approach to pricing perpetual American options. ASTIN Bull. 24, 195-220.

[14] Hilberink, B. And Rogers, L. C. G. (2002). Optimal capital structure and endogeneous default. Finance Stoch. 6, 237-263.

[15] Kyprianou, A. E. (2006). Introductory Lectures on Fluctuations of Lévy Processes with Applications. Springer, Berlin.

[16] Kyprianou, A. E. ANd Palmowski, Z. (2005). A martingale review of some fluctuation theory for spectrally negative Levy processes. In Séminaire de Probabilités XXXVIII (Lecture Notes Math. 1857), Springer, Berlin, pp. 16-29.

[17] Kyprianou, A. E. And Surya, B. A. (2007). Principles of smooth and continuous fit in the determination of endogenous bankruptcy levels. Finance Stoch. 11, 131-152.

[18] Lambert, A. (2000). Completely asymmetric Lévy processes confined in a finite interval. Ann. Inst. H. Poincaré Prob. Statist. 2, 251-274.

[19] Leland, H. E. AND Toft, K. B. (1996). Optimal capital structure, endogeneous bankruptcy, and the term structure of credit spreads. J. Finance 51, 987-1019.

[20] Oberhettinger, F. (1973). Fourier Transforms of Distributions and Their Inverses; A Collection of Tables. Academic Press, New York.

[21] Pistorius, M. R. (2004). On exit and ergodicity of the spectrally one-sided Lévy process reflected at its infimum. J. Theoret. Prob. 17, 183-220.

[22] Rogers, L. C. G. (2000). Evaluating first-passage probabilities for spectrally one-sided Lévy processes. J. Appl. Prob. 37, 1173-1180.

[23] Yang, H. And Zhang, L. (2001). Spectrally negative Lévy processes with applications in risk theory. Adv. Appl. Prob. 33, 281-291. 